\title{
PHONON DEFORMATION POTENTIALS FROM RAMAN MEASUREMENTS ON SEMICONDUCTOR MEMBRANES
}

W. Trzeciakowski ${ }^{a, b}$, J. Martinez-Pastor ${ }^{b}$ and G. Martinez-Criado ${ }^{b}$

${ }^{a}$ High Pressure Research Center, Polish Academy of Sciences

Sokołowska 29, 01-142 Warszawa, Poland

${ }^{b}$ Departamento de Fisica Aplicada, Universidad de Valencia 46100 Burjassot (Valencia), Spain

It is shown that the phonon deformation potentials in semiconductors can be determined by Raman scattering on hydrostatically and biaxially deformed samples. The complete data includes biaxial deformation in the $(100),(111)$, and (110) planes. Biaxial strain is applied to the sample using the recently developed membrane method. The phonon displacement under biaxial strain can be directly obtained from Raman measurements on a single membrane provided we determine the strain from the splitting of the band gap using e.g. the photoreflectance technique. Alternatively, the ratios of different phonon shifts can supply the necessary information. The method is illustrated with experimental results for $\mathrm{GaP}$.

PACS numbers: 78.30.Fs, 78.20.Hp, 63.20.Ry

\section{Introduction}

Recent progress in heteroepitaxial growth techniques has increased the need for measuring the strain present in different layers of the microstructure. Apart from X-ray diffraction (which may be insensitive to very thin layers) the most common technique used for this purpose is the Raman scattering. The method consists in determining the shifts of the optical phonons from their unstrained positions and then in calculating the strain using the so-called phonon deformation potentials (PDP). The PDP are the components of the fourth rank tensor relating the changes of the spring constants of the lattice and the deformation tensor. They are due to the anharmonicity of the lattice vibrations. In crystals of zinc-blende or diamond symmetry there are three independent PDP for each optical phonon. It has been initially assumed that these parameters are identical for $\mathrm{TO}$ and for LO phonons but the experiments proved the contrary. Therefore, in principle, the optical-phonon shifts due to an arbitrary deformation are described by six PDP.

Since the pioneering work on $\mathrm{Si}[1]$ there have been many measurements of PDP in practically all available semiconductor materials [2-6]. A combination of 
two PDP yields the so-called Gruneisen parameter determining the phonon shifts due to hydrostatic pressure. This parameter is known to a higher accuracy than other combinations of PDP which have been determined from uniaxial measurements. The first reason is that hydrostatic measurements in the diamond-anvil cell can be performed up to the phase transition and the deformation range (i.e. also the phonon shift) is typically an order of magnitude higher than in uniaxial experiments. The value of stress is also determined more precisely than in uniaxial studies where the stress at the surface and inside the sample may be different plus the possibility of microcracks or other defects which can release the strain.

The non-hydrostatic deformation lowers the symmetry of the lattice and removes the degeneracy of the triply degenerate optical phonons. For the uniaxial stress along high-symmetry directions (like (100) or (111)) we usually get one phonon frequency corresponding to vibrations along the strain axis and two degenerate frequencies in the directions perpendicular to strain. For the lower-symmetry directions of uniaxial strain (e.g. along the (110)) we obtain three different frequencies corresponding to three inequivalent directions. In order to determine the complete set of PDP it is essential to measure as many strain-split components as possible. For uniaxial studies it turned out that it is better to work in the region of transparency of the sample (below the band gap) because this allows for penetrating the depth of the sample and also for coupling to more phonon modes than the backscattering geometry used in the above-band-gap region.

Here we will show that using the Gruneisen parameters (obtained from hydrostatic measurements) and the biaxial-deformation results on samples with (100), (111), and (110) orientation we can obtain the full set of PDP for LO and TO phonons.

\section{Biaxial method - theoretical considerations}

Let us assume that we apply the in-plane deformation $\varepsilon$ to the zinc-blende crystal. Using the elasticity theory it is straightforward to determine the full deformation tensor in crystallographic axes. Phonon shifts due to such deformation can then be determined from the $3 \times 3$ secular equation (see e.g. [2]). For the (100) biaxial strain each optical phonon splits into a singlet $\Delta \omega_{1}$ (vibrations parallel to (100)) and a doublet $\Delta \omega_{2}$ (vibrations perpendicular to (100)):

$$
\begin{aligned}
& \frac{\Delta \omega_{1}}{\omega_{0}}=\left(-3 \gamma-C_{1} P\right) \varepsilon, \\
& \frac{\Delta \omega_{2}}{\omega_{0}}=\left(-3 \gamma-C_{1} Q\right) \varepsilon,
\end{aligned}
$$

where $P, Q$ and $R$ are the dimensionless phonon deformation potentials, $\gamma$ is the Gruneisen constant equal to $-(P+2 Q) / 6$ and $C_{1}=\left(C_{11}+2 C_{12}\right) / 2 C_{11}\left(C_{i j}\right.$ are the elastic stiffness constants). Similarly, for the (111) biaxial strain

$$
\begin{aligned}
& \frac{\Delta \omega_{1}}{\omega_{0}}=\left(-3 \gamma-C_{2} R\right) \varepsilon, \\
& \frac{\Delta \omega_{2}}{\omega_{0}}=\left(-3 \gamma+\frac{1}{2} C_{2} R\right) \varepsilon,
\end{aligned}
$$


where $C_{2}=\left(C_{11}+2 C_{12}\right) / 2 C_{44}$. Finally, for the (110) biaxial strain the phonon frequency splits into three components corresponding to vibrations along three inequivalent directions $((001),(1,-1,0)$, and $(110))$ :

$$
\begin{aligned}
& \frac{\Delta \omega_{1}}{\omega_{0}}=\left(-3 \gamma-C_{3} Q\right) \varepsilon, \\
& \frac{\Delta \omega_{2}}{\omega_{0}}=\left[-3 \gamma-\frac{1}{2} C_{3}(P+Q-2 R)\right] \varepsilon, \\
& \frac{\Delta \omega_{3}}{\omega_{0}}=\left[-3 \gamma-\frac{1}{2} C_{3}(P+Q+2 R)\right] \varepsilon,
\end{aligned}
$$

with $C_{3}=\left(C_{11}+2 C_{12}\right) /\left(2 C_{44}+C_{11}+C_{12}\right)$. In the above equations $\Delta \omega_{i}$ are different for each orientation of biaxial strain $\varepsilon$ and are different for the LO and TO phonons (because $P, Q$, and $R$ are different). However, we prefer not to add too many indices to the symbols.

The observation of the strain-split phonon lines in Raman scattering depends on the scattering geometry. For backscattering at normal incidence from the (100) surface we can only observe $\omega_{1}$ component of the LO phonon. In the case of (111) surface we can see $\omega_{1}$ component of the LO phonon and the $\omega_{2}$ component of the TO phonon. In the case of (110) surface $\omega_{1}$ and $\omega_{2}$ components of the TO phonon can be observed. However, for tilted incidence in addition to the above we can see the $\omega_{2}$ component of the TO phonon in the $(100)$ case and the $\omega_{3}$ component of the LO phonon in the (110) case. As a matter of fact, at tilted incidence the phonon frequencies are not "pure" $\omega_{1}, \omega_{2}$ and $\omega_{3}$ but they contain small admixtures of each other [7]. It is, however, quite simple to extract the "pure" frequencies from the observed ones. All this implies that for tilted incidence in the backscattering configuration we should be able to measure:

1) $\Delta \omega_{1}$ for LO and $\Delta \omega_{2}$ for TO in the (100) case (Eq. (1)) and in the (111) case (Eq. (2)),

2) $\Delta \omega_{1}$ and $\Delta \omega_{2}$ for TO plus $\Delta \omega_{3}$ for LO in the (110) case (Eq. (3)).

\section{Experimental implementation of the method}

We studied thin semiconductor membranes with (100), (111), and (110) surfaces deformed biaxially by the gas pressure of a few bar (the membrane method has already been described in $[8,9])$. The deficiency of that method lies in the fact that the relationship between the applied gas pressure and the in-plane deformation $\varepsilon$ is not well known (mainly because the boundary conditions at the membrane edge are not well known). Therefore we have two options. The first is to measure $\varepsilon$ in an independent experiment. At $300 \mathrm{~K}$ the most precise determination of $\varepsilon$ can be obtained from the band-gap splitting measured by photoreflectance (PR). If we could measure PR and Raman scattering on the same membrane, we could determine six PDP by studying only (100) and (111) samples (and using the Gruneisen parameters determined from hydrostatic experiments). In case we only measure Raman scattering, we can obtain the ratio of phonon shifts which does not contain $\varepsilon$. One ratio can be obtained for (100) membrane, one for (111), and two 
for (110) membrane. If we know the Gruneisen parameters from hydrostatic studies these four ratios yield four equations necessary to determine the complete set of PDP.

We tested the above procedure for $\mathrm{GaP}$, measuring the Stokes and anti-Stokes lines excited by the 457.9 and $476.5 \mathrm{~nm}$ argon laser lines [9]. All phonon components mentioned in the preceding paragraph were clearly observed and they all shifted substantially with pressure. This is contrary to the uniaxial case when some phonon lines do not shift [6] thus making it difficult to measure the ratios of the shifts. For the (100) case the ratio of the shifts for LO singlet and for TO doublet measured on different membranes was $1.6 \pm 0.1$. Equation (1) with the PDP from [6] yields 1.7 for this ratio. For the (111) case we obtained $0.96 \pm 0.1$ for the ratio of the shifts. Equation (2) with the PDP from [6] gives 0.62 in this case which implies that our values of parameter $R$ would be much lower than those of Ref. [6].

The (110) sample had a very strange behaviour which we attribute to the proximity of the E0 resonance for both laser lines which we used. All three lines which we expected to see were clearly visible but their separation was not the same for the Stokes and anti-Stokes spectra. The asymmetry of the Raman tensor close to resonance has been observed in GaAs [10] and is probably the origin of the observed effects for $(110)$ samples which have two inequivalent directions $(1,-1,0)$ and $(001)$ on the surface. The differences between the Stokes and anti-Stokes spectra have to be understood in order to interpret the results correctly.

Another problem which we found studying the (100) and (110) samples was related to their mechanical properties. In many cases the shift of the phonon frequencies was linear up to some pressure and then saturated at higher pressures. When the pressure was decreased the phonon shifts were lower than while increasing the pressure. This hysteresis was most probably due to microcracks appearing in the sample above a certain critical pressure and releasing the strain close to the membrane surface. This critical pressure was typically 2 to 4 times lower than the breakdown pressure. Therefore it was important to consider only the phonon shifts up to the critical pressure where the deformation was elastic and reversible. For the given dimensions of the membrane the critical pressure was highest for the (111) direction, lower for the (100) and the lowest for (110).

Concluding, the membrane method seems to be valid for the determination of PDP using the Raman measurements with tilted incidence from (100), (111), and (110) samples. Additional measurements of PR would help determine accurately the value of biaxial strain in the center of the membrane which would simplify the determination of PDP and allow for the direct measurement of phonon shifts with biaxial strain.

\section{Acknowledgments}

This work was supported by the Committee for Scientific Research (Poland) through grant no. 2P03B 04809. W.T. gratefully acknowledges the fellowship of the Spanish Ministry of Education and Science and the hospitality of the staff at Departamento de Fisica Aplicada, Universitat de Valencia. 


\section{References}

[1] E. Anastassakis, A. Pinczuk, E. Burstein, F.H. Pollak, M. Cardona, Solid State Commun. 8, 133 (1970).

[2] F. Cerdeira, C.J. Buchenauer, F.H. Pollak, M. Cardona, Phys. Rev. B 5, 580 (1972).

[3] E. Anastassakis, A. Cantarero, M. Cardona, Phys. Rev. B 41, 7529 (1990).

[4] P. Wickboldt, E. Anastassakis, R. Sauer, M. Cardona, Phys. Rev. B 35, 1362 (1987).

[5] E. Anastassakis, Y.S. Raptis, M. Hunermann, W. Richter, M. Cardona, Phys. Rev. $B$ 38, 7702 (1988).

[6] I. Balslev, Phys. Status Solidi B 61, 207 (1974).

[7] E. Anastassakis, J. Appl. Phys. 81, 3046 (1997).

[8] W. Trzeciakowski, T.P. Sosin, J. Phys. Chem. Solids 56, 649 (1995).

[9] W. Trzeciakowski, J. Martinez-Pastor, A. Cantarero, J. Appl. Phys. 82 (1997), accepted for publication.

[10] F. Cerdeira, E. Anastassakis, W. Kauschke, M. Cardona, Phys. Rev. Lett. 57, 3209 (1986). 